\title{
Transcriptional network constituted of CBP, Ku70, NOX2, and BAX prevents the cell death of necrosis, paraptosis, and apoptosis in human melanoma
}

\author{
Liang Ding ${ }^{1}$, Yalei Wen ${ }^{1}$, Xin Zhang ${ }^{1}$, Fang Zhao ${ }^{1}$, Kenao LV$^{2}$, Jian-hong Shi ${ }^{3}$, Shigang Shen ${ }^{4}$ and Xuefeng Pan ${ }^{1,2,4}$
}

\begin{abstract}
CREB-binding protein (CBP) is an acetyltransferase known to play multiple roles in the transcriptions of genes involving oxidative metabolism, cell cycle, DNA damage checkpoints, and cell death. In this study, CBP was found to positively regulate the expression of Ku70, and both CBP and Ku70 were found to negatively regulate the expression of NOX2, therefore, mitigating the intracellular ROS in human melanoma. Knocking down CBP or Ku70 induced necrotic and paraptotic cell death as indicated by high-level intracellular ROS, cytoplasmic vacuolization, and cell cycle arrest in the $S$ phase. In addition, chromosomal condensations were also observed in the cells proceeding necrotic and paraptotic cell death, which was found to be related to the BAX-associated intrinsic pathway of apoptotic cell death, when Ku70 was decreased either by CBP depletion or by Ku70 depletion directly. Our results, therefore, supported the idea that CBP, Ku70, BAX, and NOX2 have formed a transcriptional network in the prevention of cell death of necrosis,

paraptosis, and apoptosis in human melanoma.
\end{abstract}

\section{Introduction}

CREB-binding protein (CBP) acetyltransferase activates a number of transcription factors, including Nrf2 (nuclear factor erythroid-related factor 2$)^{1}, \mathrm{MITF}^{2}, \mathrm{NF}-\mathrm{KB}^{3}$, and AP-1 (ref. ${ }^{4}$ ). Among which, Nrf2 is involved in monitoring the reactive oxygen species (ROS) and maintaining the redox homeostasis. Nrf2 presents as Nrf2-Keap1 complex in the cytoplasm and enters the nucleus once the Keap1 is modified by ROS. Nrf2 binds to antioxidant response element (ARE) in the promoter regions of multiple genes that encode antioxidant enzymes and phase II detoxification enzymes oxidative metabolism, including heme oxygenase 1 , nicotinamide adenine dinucleotide phosphate (NADPH) quinone oxidoreductase 1 , and so forth ${ }^{5}$.

\footnotetext{
Correspondence: Xuefeng Pan (xuefengpancam@aliyun.com)

${ }^{1}$ School of Medicine, Hebei University, Baoding 071002, China

${ }^{2}$ School of Life Science, Beijing Institute of Technology, Beijin 100081, China

Full list of author information is available at the end of the article

These authors contributed equally: Liang Ding, Yalei Wen, Xin Zhang,

Fang Zhao
}

Edited by Inna Lavrik
The roles of CBP in the Nrf2-regulated gene transcriptions are twofolds, CBP facilitates the assembly of the transcription initiation complex via interacting with $\mathrm{Neh} 4$ domain and Neh5 domain in the N-terminal region of Nrf2 (ref. ${ }^{6}$ ), while it also works in acetylation of proteins, such as histones in the transcribing chromatin ${ }^{7}$.

Defective CBP has been found in human diseases, including Rubinstein-Taybi syndrome, tumors (e.g., leukemia, a variety of translocations of CBP genes have been characterized so far), and neurological disorders, such as Huntington's disease ${ }^{8}$, Alzheimer's disease ${ }^{9}$, polyglutamine diseases ${ }^{10}$, and spinal and bulbar muscular atrophy ${ }^{11}$. CBP serves as a tumor suppressor by signaling DNA damages and by regulating DNA repair factors in certain tumor conditions ${ }^{12}$. For example, in DNA repairs of double-strand break (DSB) by nonhomologous end joining (NHEJ), CBP has been found to catalyze the acetylation modifications of histones $\mathrm{H} 3$ and $\mathrm{H} 4$ at a DSB site, to promote the recruitments of Ku70/80 protein to the DSB site through synergizing with SWI/SNF chromatin remodeling complex ${ }^{13}$. In addition, the acetylation

\section{(c) The Author(s) 2021}

(c) (i) Open Access This article is licensed under a Creative Commons Attribution 4.0 International License, which permits use, sharing, adaptation, distribution and reproduction c. in any medium or format, as long as you give appropriate credit to the original author(s) and the source, provide a link to the Creative Commons license, and indicate if changes were made. The images or other third party material in this article are included in the article's Creative Commons license, unless indicated otherwise in a credit line to the material. If material is not included in the article's Creative Commons license and your intended use is not permitted by statutory regulation or exceeds the permitted use, you will need to obtain permission directly from the copyright holder. To view a copy of this license, visit http://creativecommons.org/licenses/by/4.0/. 
modification of the Ku70 by CBP can also be used in controlling its binding with BAX in initiating the mitochondrial pathway of cell apoptosis. During which, BAX is released from the Ku70-BAX complex as a result of the $\mathrm{Ku} 70$ acetylation, and the released BAX is then to initiate the mitochondrial pathway of cell apoptosis ${ }^{14-16}$. Acetylation modifications of lysine 539 and 542 in Ku70 release BAX from the Ku70-BAX complex, which leads to cell apoptosis in a BAX- and caspase-dependent fashion in neuroblastoma (NB) cells ${ }^{16}$. However, the roles of CBP in melanoma have not been clearly understood yet.

The roles of ROS in human disease conditions have been well documented ${ }^{17}$. ROS serves as the main source of intracellular redox homeostasis with strong cytotoxicity. Loss of balance between redox molecules and antioxidants in cells could exacerbate the cytotoxic effects of the ROS, leading ultimately to human diseases, including metabolic dysfunctions, neurodegeneration, chronic inflammation, cardiovascular defects, and oncogenesis ${ }^{16}$. ROS can be produced by multiple pathways of metabolism, including glycolysis, gluconeogenesis, lipid metabolism, and ATP or nitric oxide synthesis ${ }^{18}$. Among them, the oxidation of NADPH catalyzed by NADPH oxidase is the main source of ROS production ${ }^{19}$. NADPH oxidase consists of five subunits, including two membrane subunits: gp91 ${ }^{\text {phox }}(\mathrm{NOX} 2)$ and $\mathrm{p} 22^{\text {phox }}$, and three cytoplasmic subunits: $\mathrm{p} 47^{\text {phox }}, \mathrm{p} 40^{\text {phox }}$, and $\mathrm{p} 67^{\text {phox }}\left(\right.$ ref. $\left.{ }^{20}\right)$. It has been reported that epigenetic modifications, such as histone acetylation and deacetylation (histone deacetylase (HDAC)) are essential for the transcriptions of some subtypes of NADPH oxidases, such as NOX2 (ref. ${ }^{20}$ ). Decreased transcription of NOX2 mRNA by applying HDAC inhibitors has been reported in immune cells ${ }^{20}$.

In this study, we have analyzed the roles of CBP in human melanoma A375 by differentially depleting the CBP mRNA. We found that depletion of CBP mRNA upregulated the expression of NOX2 gene, encoding NOX2 NADPH oxidase, and affected the gene transcriptions of phase II detoxification enzymes via Nrf2-Keap1 pathway, resulting in the rapid elevation of intracellular ROS in melanoma cells. In addition, cytoplasmic vacuolization and cell cycle arrest in $S$ phase were also characterized, and the expression of Ku70 was also decreased. Moreover, the depletion of either CBP or Ku70 caused chromatin condensation and fragmentation, as usually seen in the intrinsic pathway of apoptotic cell death. Further, we also found that the downregulation of Ku70 gene transcription and translation was much more significant than the mitigation of acetylation modifications of the Ku70 protein by CBP depletion, showing a Ku70 dosage-dependent elevation of BAX in CBPdepleted cells. The BAX then leads to the release of pro-apototic factors, such as cytochrome $\mathrm{C}$ from the mitochondria and the activation of the caspases, resulting in the initiation of the intrinsic pathway of apoptosis. Therefore, our results adding up together indicated that CBP, Ku70, NOX2, and BAX have been made up of a transcriptional network in preventing cell death, such as paraptosis and necrosis via NOX2-ROS, and apoptosis via $\mathrm{Ku} 70-\mathrm{BAX}$-caspases in human melanoma.

\section{Results \\ Depletion of CBP and/or Ku70 inhibited cell growth and caused cell death}

To understand the roles of CBP and Ku70 in human melanoma cells, we designed and synthesized a set of CBP siRNA and Ku70 siRNA, respectively, and examined their efficiencies in knocking down the CBP mRNA and the Ku70 mRNA in human melanoma A375 cell line by realtime quantitative polymerase chain reaction (PCR). A $2^{-\triangle \triangle \mathrm{Ct}}$ method was used when quantifying the changes of the transcriptions of CBP mRNA in both the control group and the experimental groups (Fig. 1Aa). As shown in Fig. 1Aa, the CBP mRNA has successfully been downregulated in a dosage-dependent manner after the CBP siRNA was transfected into cells by different amounts (Fig. 1Aa).The depletion efficiencies were found to be 20,40 , and $70 \%$ when transfections were done by 1 , 2 , and $3 \mathrm{fmol} /$ cell $(P<0.05)$. By contrast, transfections using negative control siRNA (NC group) failed to decrease the CBP mRNA in the control group $(P>0.05)$.

The effects of CBP depletion on cell proliferation were then analyzed using a CCK- 8 assay. The CCK- 8 results are presented in Fig. $1 \mathrm{Ab}$. As it can be seen in Fig. $1 \mathrm{Ab}$, depletion of CBP inhibited the cell proliferation of the A375 cells in a siRNA dose-dependent manner. The more the depletion of the CBP mRNA, the more inhibiting effects on cell proliferation of the A375 cells were seen (Fig. 1Ab). By contrast, no significant differences in proliferation inhibition can be noticed between the NC group and the control group (Fig. 1Ab).

Similarly, the Ku70 mRNA in the control group and in the cells differentially depleting Ku70 were also determined by real-time quantitative PCR (Fig. 1Ac). Approximate depletion efficiencies of 30,70 , and $80 \%$ were found to be actualized when 1, 2, and $3 \mathrm{fmol} / \mathrm{cell}$ of Ku70 siRNA were used in the transfection manipulations, respectively $(P<0.05$; Fig. 1 Ac). The CCK- 8 proliferation analysis with the cells depleting Ku70 mRNA are presented in Fig. 1Ad, as shown in Fig. 1Ad, depletion of Ku70 mRNA also inhibited the cell proliferation of the A375 cells in a siRNA dose-dependent manner (Fig. 1Ad).

We then compared the effects of co-transfections of CBP siRNA (70\% depletion efficiency) and Ku70 siRNA (70\% depletion efficiency) on cell proliferation. The depletion of CBP mRNA by $70 \%$ and the depletion of Ku70 mRNA by $70 \%$ were actualized by using $3 \mathrm{fmol} /$ cell of CBP siRNA and $2 \mathrm{fmol} / \mathrm{cell}$ of Ku70 siRNA, 


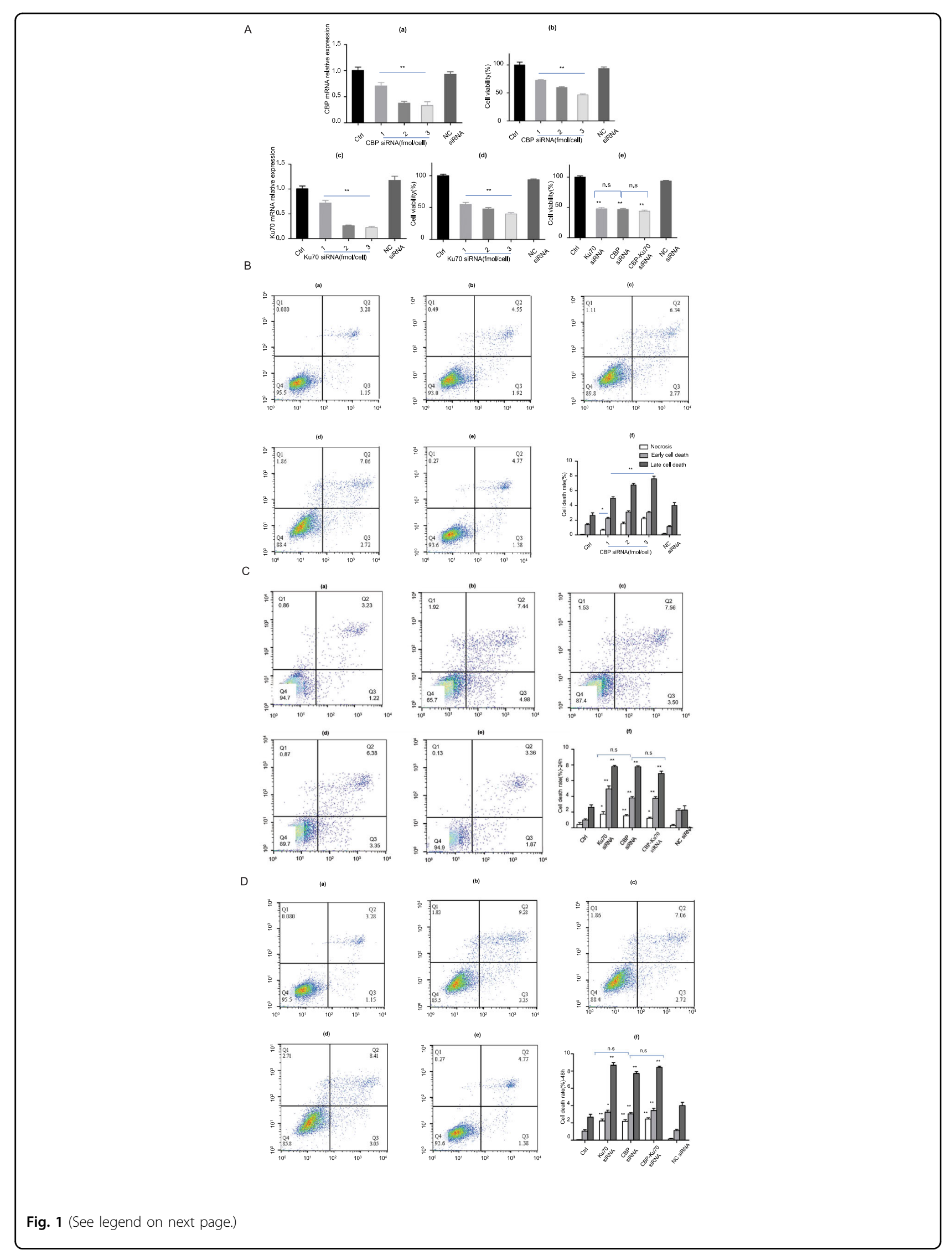


(see figure on previous page)

Fig. 1 Depletion of CBP and/or Ku70 inhibited cell proliferation and caused cell death. A (a) CBP mRNA expression level in human melanoma cells line A375 cells; (b) CCK-8 assay on cell proliferation after transfection CBP siRNA; (c) Ku70 mRNA expression level in human melanoma cells line A375 cells; (d) CCK-8 assay on cell proliferation after transfection Ku70 siRNA; (e) CCK-8 assay on cell proliferation after transfection CBP siRNA and/or Ku70 siRNA. B Cell death caused by the depletion of CBP in human melanoma A375 cells in 48 h. (a) Control group. (b) CBP siRNA (1 fmol/cell) group. (c) CBP siRNA (2 fmol/cell) group. (d) CBP siRNA (3 fmol/cell) group. (e) NC siRNA group; (f) the statistical analysis of the cell death. C Effects on A375 cell death by co-transfection of CBP siRNA and Ku70 siRNA (24 h). (a) Control group; (b) Ku70 siRNA (70\% depletion) group; (c) CBP siRNA (70\% depletion) group. (d) Co-transfections of the CBP siRNA and the Ku70 siRNA (60\% depletion for CBP and Ku70, respectively); (e) NC siRNA group; (f) the statistical analysis of the cell death rate of A375 cells. D Effects on A375 cell death by co-transfection of CBP siRNA and Ku70 siRNA (48 h). (a) Control group; (b) Ku70 siRNA (70\% depletion) group; (c) CBP siRNA (70\% depletion) group; (d) co-transfections of CBP siRNA and Ku70 siRNA (60\% depletion for CBP and Ku70, respectively); (e) NC siRNA group; (f) the statistical analysis of the cell death rate of A375 cells. Data in all bars are expressed as mean \pm SEM $(n=3)$. ${ }^{*} P<0.05,{ }^{*} P<0.01,{ }^{\#} P<0.05,{ }^{\# \#} P<0.01$, and n.s denotes $P>0.05$, when compared with CBP siRNA (by $70 \%$ depletion) group.

respectively $(P<0.05$, Fig. 1 Aa, Ac). The effects of cotransfections on cell proliferation are presented in Fig. 1Ae. As it can be seen in Fig. 1Ae, the proliferation of A375 cells were found to be significantly inhibited in the CBP siRNA (70\% depletion) group, the Ku70 siRNA (70\% depletion) group, and the CBP-Ku70 co-transfection (each with a depletion of $60 \%$ by PCR quantification), respectively (Fig. 1Ae). No significant differences in the inhibition on A375 cell proliferation between the groups of Ku70 siRNA, the CBP siRNA, and the cotransfection siRNA were noticed (Fig. 1Ae), suggesting that the depletion of CBP and Ku70 individually or simultaneously affected the cell proliferation of A375 cells in a similar fashion.

To understand the mechanism underpinning the cell proliferation inhibition by depleting CBP and Ku70, we have analyzed the cell death induced by the depletion of CBP by flow cytometry, we found a positive correlation between the necrosis, early cell death, and late cell death and the differential depletion of CBP (Fig. 1B). In addition, we also compared the cell death induced by depleting CBP and Ku70, and found no significant difference between the two sets of analysis (Fig. 1C, D), which is in good agreement with the Cell Counting Kit-8 (CCK-8) analysis.

\section{Depletion of CBP and/or Ku70 caused significant cytoplasmic vacuolization and ROS accumulation}

The morphological changes of the cells affected by the transfections of either CBP siRNA (by 70\% depletion) or Ku70 siRNA (by 70\% depletion) were also observed under a light microscope (Fig. 2A). As shown in Fig. 2A, significant cytoplasmic vacuolization was found to be associated with the proliferation of A375 cells depleting CBP mRNA and/or Ku70 mRNA (Fig. 2A).

To understand the cause underpinning the cytoplasmic vacuolization, we have further examined the alterations of the intracellular ROS in the cells depleting CBP and Ku70 by flow cytometry (Fig. 2B). As shown in Fig. 2B, a significant elevation in intracellular ROS levels was found to be associated with the CBP depletion by a siRNA dosagedependent manner (Fig. 2B). We then further compared the ROS production in the cells individually depleting CBP (by 70\% depletion), Ku70 (by 70\% depletion), and simultaneously depleting both CBP (by $60 \%$ depletion) and Ku70 (by $60 \%$ depletion; Fig. 2C). The results are presented in Fig. 2C. As it can be seen in Fig. 2C, the intracellular ROS in A375 cells depleting Ku70 was indeed elevated (Fig. 2C). But it was elevated slightly less than the intracellular ROS in the cells depleting CBP (Fig. 2C). Interestingly, the intracellular ROS in the cells simultaneously depleting Ku70 and CBP showed similar elevation to the intracellular ROS in the cells depleting CBP mRNA only (Fig. 2C), suggesting that CBP and Ku70 may work together in repressing the intracellular ROS production (Fig. 2C).

Paraptotic cell death and necrotic cell death do not show apoptotic characteristics of pyknosis, DNA fragmentation, chromatin condensations, and caspase activations $^{21}$, but share common features of cytoplasmic vacuolization $^{22}$. The cytoplasmic vacuolization shared by them could be distinguished by a different response to actinomycin D. Actinomycin D inhibits gene transcription by binding DNA at the transcription initiation complex and preventing elongation of RNA by RNA polymerase, which is expected to mitigate the cytoplasmic vacuolization in the parapototic cell death. In this work, we found that the cytoplasmic vacuolization associated with ROS accumulation can be decreased by actinomycin D (Fig. 2D), suggesting that the cytoplasmic vacuolization associated with the CBP depletion or the Ku70 depletion was at least an indication of the presence of paraptotic cell death (Fig. 2D).

\section{Increased ROS by CBP depletion was due partially to the malfunctions of the transcriptions of the genes in the Nrf2-Keap1 pathway}

To understand if the increased ROS may or may not be due to the failure of CBP-associated transcriptions of genes encoding intracellular antioxidant enzymes and the 


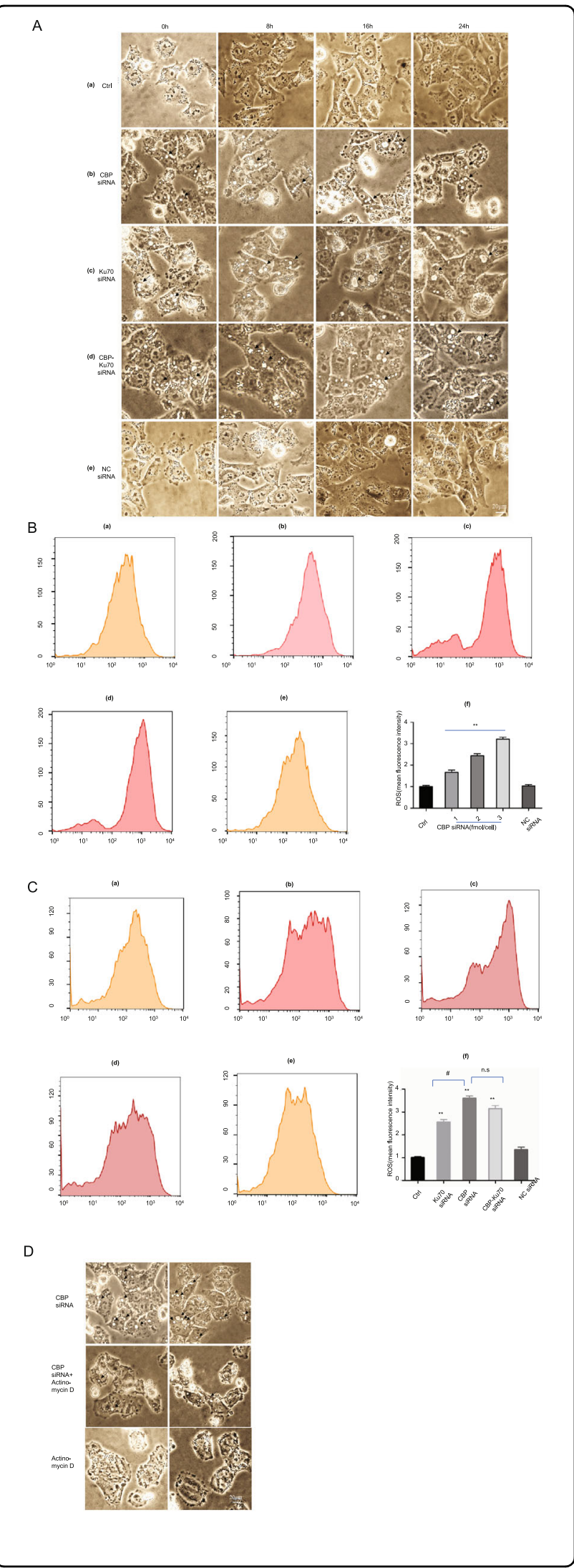

Fig. 2 Depletion of CBP and/or Ku70 induced cytoplasmic vacuolization and elevated the intracellular ROS. A Cytoplasmic vacuolization (cell morphology under a light microscope ( $\times 400)$ ). (a) Control group; (b) CBP siRNA (70\% depletion) group; (c) Ku70 siRNA (70\% depletion) group; (d) co-transfections of CBP siRNA and Ku70 siRNA (60\% depletion for CBP and Ku70, respectively); (e) NC siRNA group. Cytoplasmic vacuolization appeared $\mathrm{O}$ h after CBP siRNA transfection (black triangle); B ROS generation was measured by the DCF fluorescence intensity. (a) Control group. (b) CBP siRNA (1 fmol/ cell) group. (c) CBP siRNA (2 fmol/cell) group. (d) CBP siRNA (3 fmol/ cell) group. (e) NC siRNA group. (f) Quantitative analysis of ROS generation. C Effects of co-transfection of CBP siRNA and Ku70 siRNA on the ROS accumulation in A375 cells. (a) Control group. (b) Ku70 siRNA (70\% depletion) group. (c) CBP siRNA (70\% depletion) group. (d) Co-transfections of CBP siRNA and Ku70 siRNA (60\% depletion for CBP and Ku70, respectively) group. (e) NC siRNA group; (f) quantitative analysis of ROS generation. Data as shown in the bars are expressed as mean \pm SEM $(n=3) .{ }^{*} P<0.05, P<0.01,{ }^{\sharp} P<0.05$,

${ }^{\# \# P} P<0.01$, and n.s denotes $P>0.05$, when compared with CBP siRNA (70\% efficiency) group. D The cytoplasmic vacuolization induced by ROS accumulation is actinomycin D dependent in human melanoma A375. CBP siRNA (70\% depletion) group. CBP siRNA (70\% depletion) and actinomycin D $(5 \mu \mathrm{g} / \mathrm{ml})$ group. Actinomycin $D(5 \mu \mathrm{g} / \mathrm{ml})$ group.

phase II detoxification enzymes in the Nrf2-Keap1 pathway $^{23,24}$. CBP and Nrf2 siRNAs were co-transfected with the melanoma cells by a dose roughly equal to $70 \%$ depletion of either CBP mRNA or Nrf2 mRNA. The yields of ROS in the cells depleting either CBP mRNA, Nrf2 mRNA alone, or depleting both CBP mRNA and Nrf2 mRNA were measured by flow cytometry, and the results are presented in Fig. 3. As it can be seen in Fig. 3, the ROS levels in the cells depleting CBP and in the cells depleting Nrf2 mRNA were similar, no significant differences can be seen between them (Fig. 3). However, a synthetic effect of the ROS accumulation by simultaneously depleting both CBP mRNA and Nrf2 mRNA can be clearly seen, when compared with the ROS accumulated in the cells depleting either CBP mRNA or Nrf2 mRNA individually, suggesting that the enhancement of ROS level by depleting CBP mRNA was not purely due to the failure of CBP functions in the gene transcriptions of the intracellular antioxidant enzymes and the phase II detoxification enzymes in the Nrf2-Keap1 pathway, rather our results suggested that the depletion of CBP mRNA may also enhance the ROS production in an Nrf2-Keap1 pathwayindependent manner (Fig. 3).

\section{Depletion of either CBP or Ku70 increased expression of NOX2}

To further understand the cause of the elevated ROS production in the cells depleting CBP, we then carried out the reverse transcription-quantitative PCR (RT-qPCR) quantification of the NOX2 mRNA in the A375 cells 
(a)

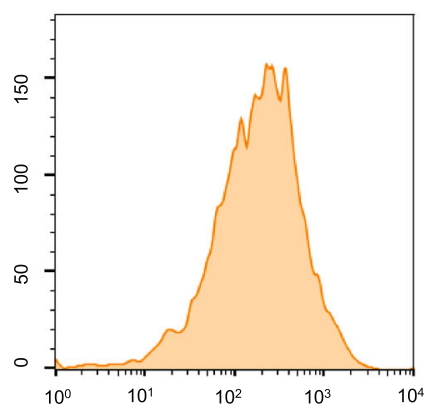

(d)

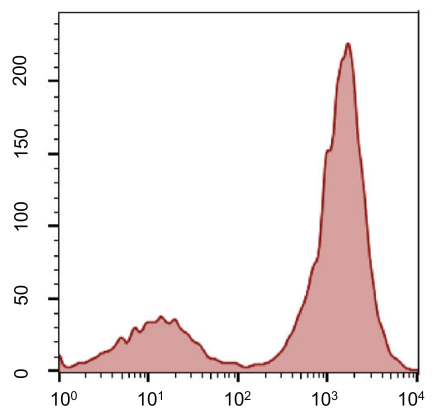

(b)

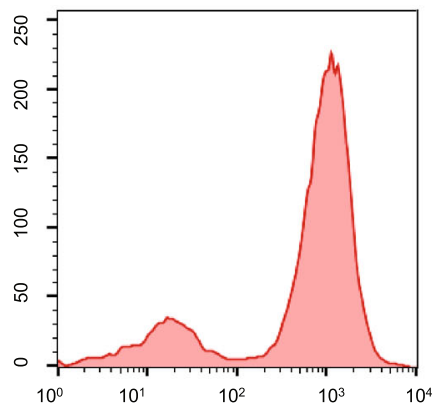

(e)

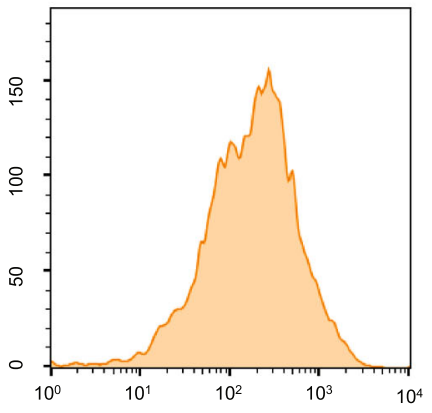

(c)
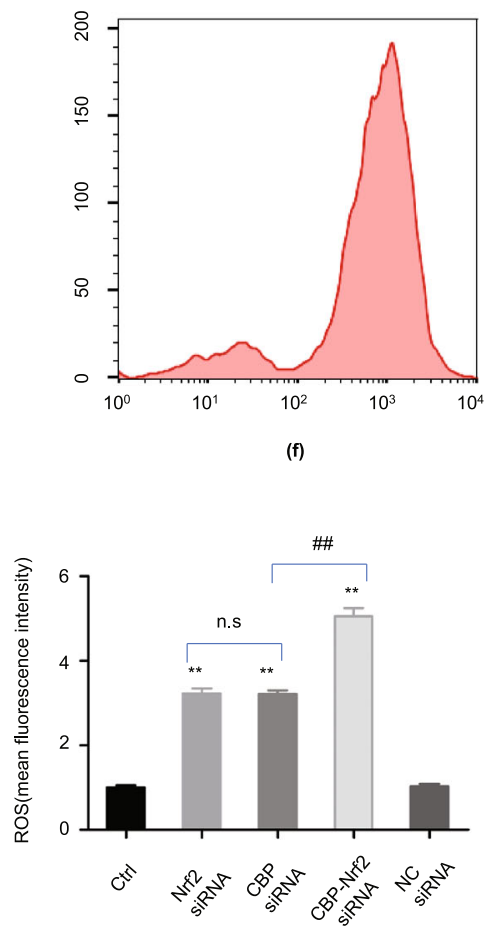

Fig. 3 Effects of co-transfection of CBP siRNA and Nrf2 siRNA on the ROS accumulation in A375 cells. a Control group; b Nrf2 siRNA(70\% depletion); c CBP siRNA(70\% depletion); $\mathbf{d}$ co-transfections of CBP siRNA and Nrf2 siRNA (the dose of siRNA applied equals to 70\% depletion of CBP and Nrf2, respectively); e NC siRNA group; $\mathbf{f}$ measurements of the ROS by the DCF fluorescence intensity. Data as shown in the figures were expressed as mean $\pm \operatorname{SEM}(n=3) .{ }^{*} P<0.05,{ }^{* *} P<0.01,{ }^{\#} P<0.05,{ }^{\# \#} P<0.01$, and n.s denotes $P>0.05$.

depleting CBP (Fig. 4a). As shown in Fig. 4a, differential depletion of CBP upregulated the gene transcription of NOX2 in a dose-dependent manner (Fig. 4a).

To further investigate the mechanism of ROS accumulation in Ku70-depleted A375 cells, we also performed RT-qPCR analysis on the transcription of NOX2 mRNA in the cells depleting Ku70 (Fig. 4b). As shown in Fig. 4b, NOX2 mRNA expression was indeed upregulated in the Ku70-depleted cells. Interestingly, we found that the upregulation of NOX2 in the cells depleting CBP or Ku70 was similar to each other (Fig. 4a, b), suggesting that CBP and Ku70 are presumably playing similar roles in the transcription of the NOX2 gene. Finally, we also confirmed the increase in the protein level of NOX2 by western blotting (Fig. 4c, d). As shown in Fig. 4c, d, the expressions of NOX2 protein were indeed increased (Fig. 4c, d).

\section{Depletion of CBP/Ku70 induced chromatin condensation}

Since we observed a significant increase in the late cell death are associated with the cells depleting either CBP and/or Ku70, we then attempted to know if apoptotic cell death was also existing. To this end, we stained the cells depleting CBP and/or Ku70 (as aforementioned) with DAPI to look at the possible chromatin condensation known to be associated with apoptotic cell death. We visualized the DAPI-stained nucleus under a confocal microscope (Fig. 5). As shown in Fig. 5, vigorous chromatin condensations were indeed seen in the cells depleting either CBP or Ku70 individually, or depleting both CBP and Ku70 simultaneously (Fig. 5), suggesting that apoptotic cell death was indeed being induced by the depletion of CBP and/or Ku70 (Fig. 5).

\section{Depletion of CBP downregulated the expression of Ku70 and caused apoptosis}

To understand the possible relations between CBP and Ku70 in the elevation of intracellular ROS, we then determined the expression of Ku70 in the cells depleting $\mathrm{CBP}, \mathrm{Ku} 70$, and $\mathrm{CBP}-\mathrm{Ku} 70$, respectively. The results are presented in Fig. 6. As shown in Fig. 6, the depletion of the Ku70 alone didn't affect the transcription of CBP mRNA (Fig. 6a), while the depletion of CBP did significantly downregulate both the mRNA and the protein of Ku70 (Fig. 6b-d), indicating that CBP regulates the expression of $\mathrm{Ku} 70$, not vice versa. Further, we also detected the acetylation modification of the Ku70 in the cells depleting CBP (Fig. 6e), we found that the acetylated Ku70 was reduced in the cells depleting CBP mRNA (Fig. 6e), but the reduction was rather similar to that in 
(a)

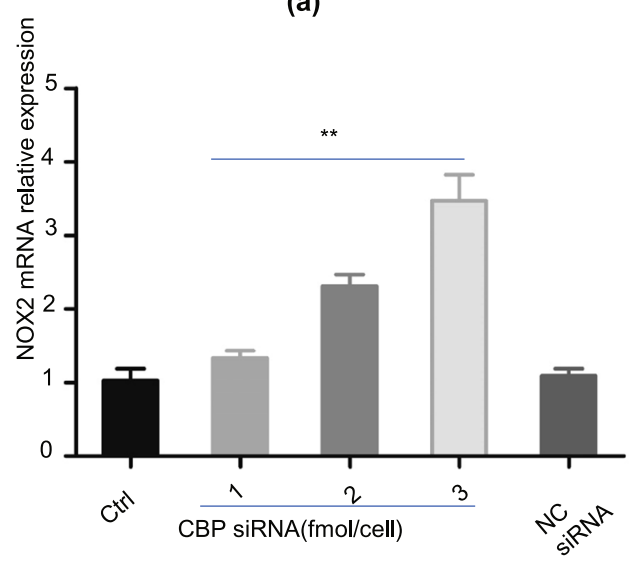

(c)

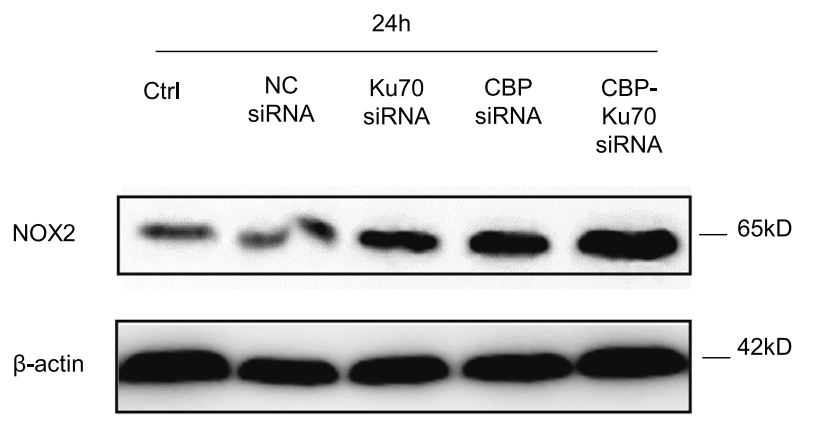

(b)

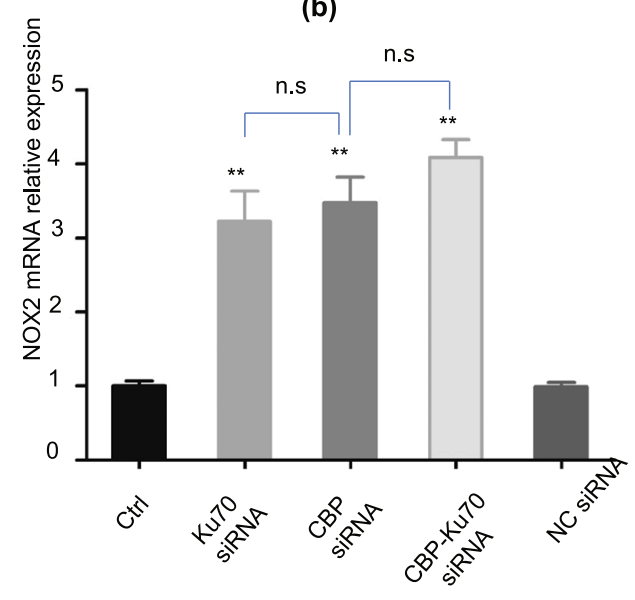

(d)

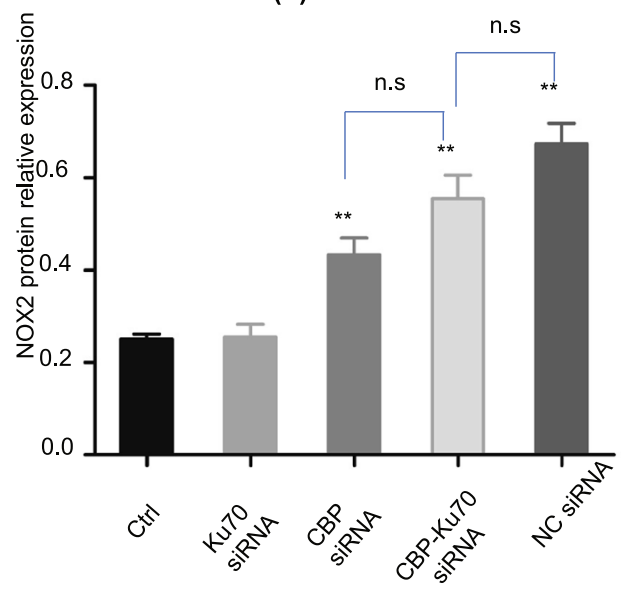

Fig. 4 Depletion of CBP and/or Ku70 enhanced the expression of NOX2. a The NOX2 mRNA level evaluated by RT-PCR; $\mathbf{b}$ the transcription of NOX2 when depleting Ku70, and simultaneously depleting both CBP and Ku70; $\mathbf{c}$ increased NOX2 protein in the cells depleting CBP and/or Ku70 measured by western blotting (the expression was compared to human $\beta$-actin as a gene for normalization); $\mathbf{d}$ The NOX2 protein level evaluated using western blotting, when depleting CBP or Ku70 individually or simultaneously. Data as shown in the figures were expressed as mean \pm SEM $(n=3) .{ }^{*} P<0.05,{ }^{*} P<0.01,{ }^{\#} P<0.05,{ }^{\# \#} P<0.01$, and n.s denotes $P>0.05$.

the cells depleting Ku70 mRNA individually (Fig. 6e), suggesting that the reduction of the acetylated Ku70 in the cells depleting CBP was mainly dependent on the reduced expression of $\mathrm{Ku} 70$ rather than the reduced acetylation capacity of the CBP (Fig. 6e). We then further analyzed the BAX and caspase- 3 in the cells depleting CBP (Fig. 6f, g). We found that both BAX expression (Fig. 6f) and caspase-3 activity (Fig. 6g) were increased. These results suggested that CBP has played dual roles by regulating the $\mathrm{Ku} 70$ expression and by the acetylation modification of $\mathrm{Ku} 70$.

\section{Depletion of CBP and Ku70 arrested cell cycle in S phase}

To investigate if the depletion of CBP and/or Ku70 affected the cell cycle of the melanoma cells, we have further analyzed the cell cycle of the melanoma cells depleting CBP and/or Ku70 by flow cytometry. The results are presented in Fig. 7a-f. As shown in Fig. 7a-f, the cell numbers of A375 cells depleting either CBP or Ku70 showed no significant difference to those of the NC siRNA and control groups in the G2/M phase of the cell cycle. However, numbers of S phase cells in the CBP and/ or Ku70-depleted groups were significantly increased compared to those in the NC siRNA group. These results clearly indicated that depletion of either CBP or Ku70 in melanoma cells arrested the cell cycle in the $\mathrm{S}$ phase, conceivably due to the elevated ROS made by the increased NOX2 (Fig. 7a-f).

\section{Discussion}

Human melanoma is a highly invasive and fatal disease, intervention as early as possible has thought to be 


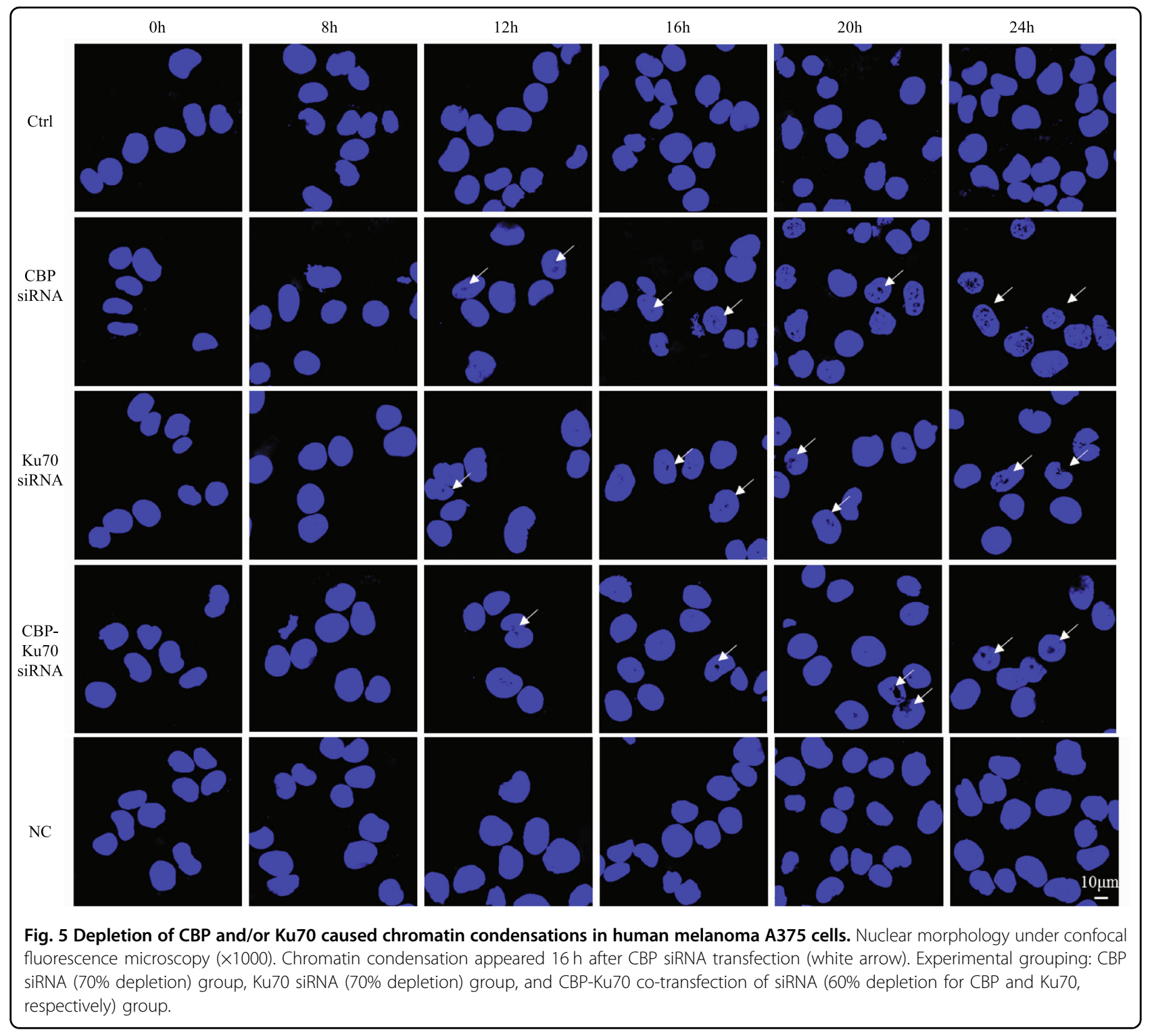

essential for an ideal prognosis ${ }^{25}$. It has been well documented that the proliferation of melanoma cells may be challenged by a high level of ROS produced by multiple pathways of metabolic processes, including mitochondria, melanosomes, NOX, and $\mathrm{NOS}^{26}$. ROS could affect melanoma proliferation in dual ways, excessive intracellular ROS may increase DNA damage and chromosomal degradation, leading to genetic instability, or activate antitumor signaling pathways, initiating oxidative stressinduced tumor cell death, or promote tumor reductive signal transduction and enhance cell proliferation and survival $^{27}$.

The Nrf2-Keap1 pathway is found to be a key mechanism of the ROS monitor. Of which, CBP worked with Nrf2 in transcribing the genes encoding the intracellular antioxidant enzymes and the phase II detoxification enzymes ${ }^{28-30}$ When the ROS level was raised, Nrf2 disassociates with Keap1 and enters the nucleus to bind its specific ligand Maf, forming a heterodimer, Nrf2-Maf. This heterodimer then binds the ARE with the assistance of CBP to activate gene transcriptions of antioxidative enzymes and phase II detoxification enzymes as aforementioned $^{23}$.

In this study, we have found that CBP has not only been used in the transcriptional activation of the Nrf2 target genes through interacting with the CREB bound in the CRE cis-acting element (cAMP response element; as previously reported), but also played opposite roles in repressing the expression of NOX2 and in enhancing the expression of Ku70. Depletion of CBP promoted the NOX2 expression while downregulated the Ku70 expression, leading to significant cell death showing server 
(a)

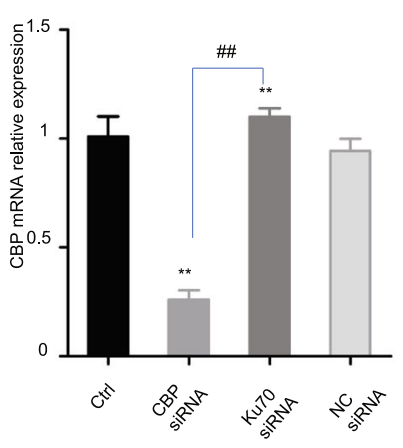

(b)

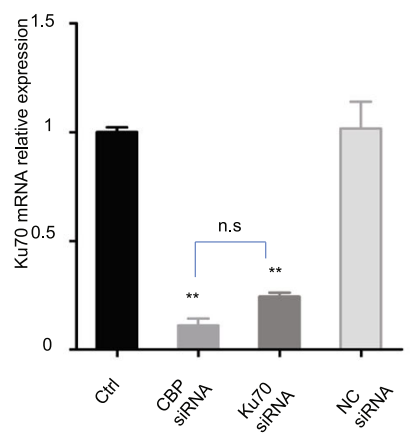

(c)

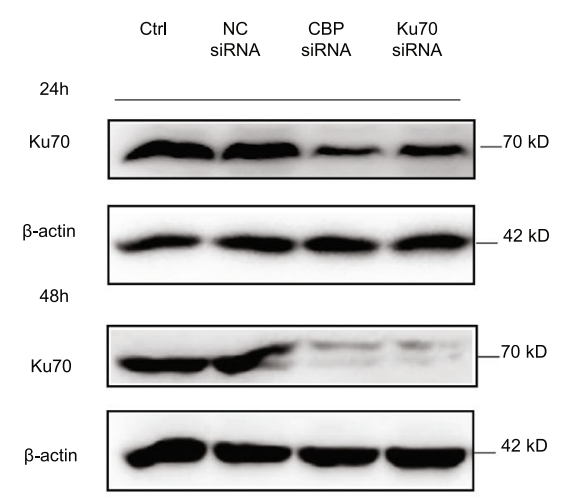

(e)
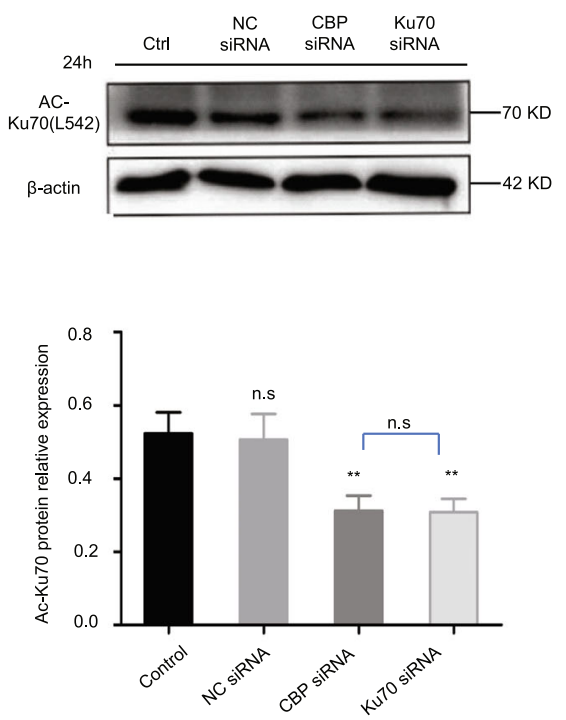

(g)

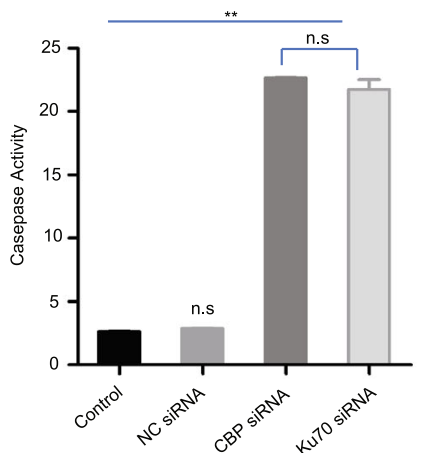

Fig. 6 Depletion of CBP downregulated the expression and acetylation modification of Ku70. a Changes in CBP mRNA in melanoma cells depleting either Ku70 or CBP, respectively; b changes in Ku70 mRNA in melanoma cells depleting either Ku70 or CBP, respectively; c changes in the level of Ku70 protein molecules in melanoma cells depleting either Ku70 or CBP for 24 and 48 h, respectively; d quantifications of Ku70 protein levels in melanoma cells when depleting either Ku70 or CBP based upon the western blotting in c; e western blotting analysis on the Ku70(L542) (modified by acetylation at L542) in melanoma cells when depleting either Ku70 or CBP for $24 \mathrm{~h}$; f Western blotting analysis on the BAX in melanoma cells when depleting either Ku70 or CBP for $24 \mathrm{~h} ; \mathbf{g}$ the elevated caspase-3 activity when depleting either CBP or Ku70. The caspase-3 activity was measured using the chromogenic substrate AcDEVD-pNA when the corresponding depletion was conducted for $24 \mathrm{~h}$. The bar represents the increase of the absorbance of pNA produced as a function of caspase-3 cleavages. Data as shown in the figures were expressed as mean $\pm \operatorname{SEM}(n=3) .{ }^{*} P<0.05,{ }^{* *} P<0.01,{ }^{*} P<0.05,{ }^{\# \#} P<0.01$, and n.s denotes $P>0.05$. 

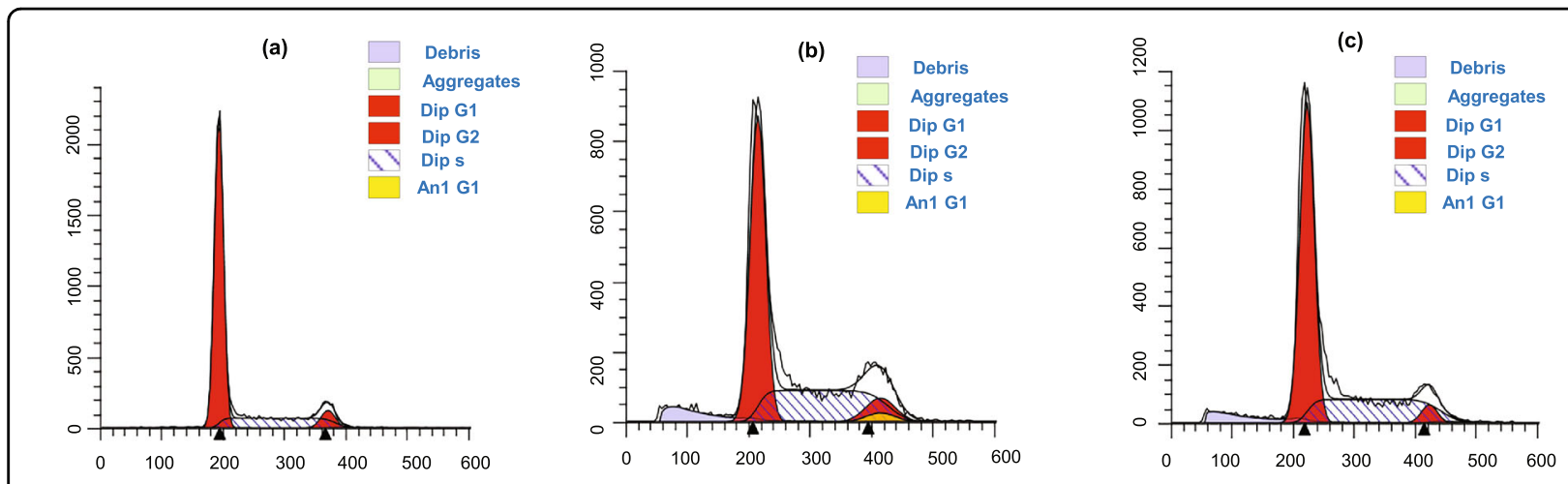

(d)

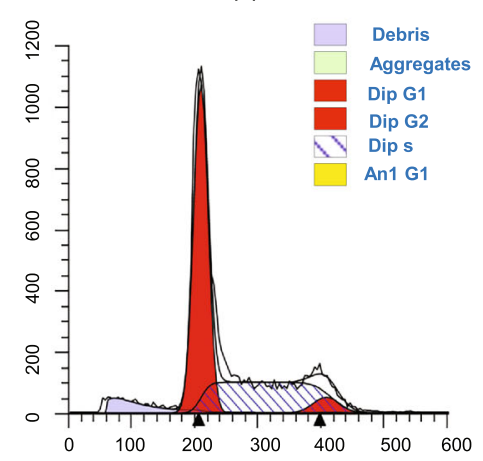

(e)

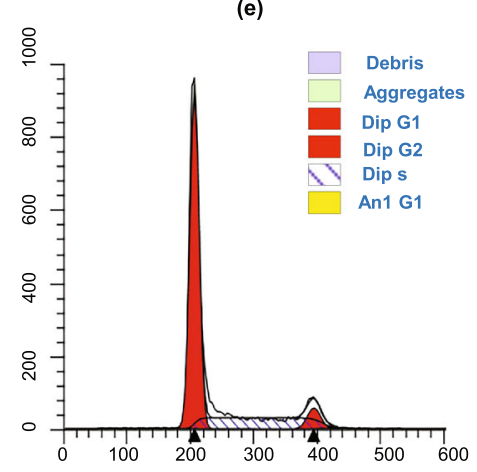

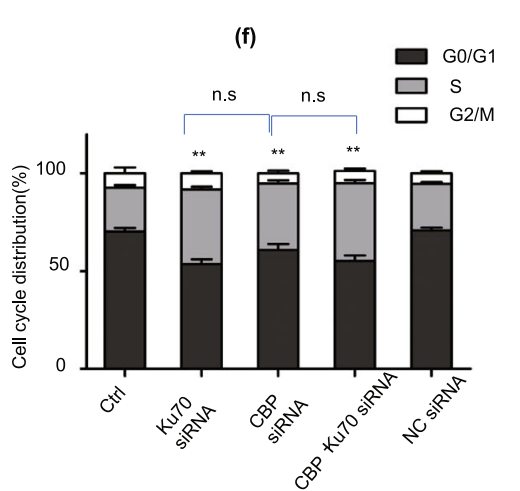

Fig. 7 Depletion of CBP and/or Ku70 caused cell cycle arrest. a Control group. b Ku70 siRNA (70\% depletion) group; c CBP siRNA (70\% depletion) group; d co-transfections of CBP siRNA and Ku70 siRNA (60\% depletion for CBP and Ku70, respectively). e NC siRNA group; f cell cycle of different groups of A375 cells. Data as shown in the figures were expressed as mean $\pm \operatorname{SEM}(n=3) .{ }^{*} P<0.05,{ }^{* *} P<0.01,{ }^{\#} P<0.05$, ${ }^{\# \#} P<0.01$, and n.s denotes $P>0.05$.

cytoplasmic vacuolization, increased ROS accumulation, cell cycle arrest in the $\mathrm{S}$ phase, and chromosomal fragmentation in melanoma cells.

A working model illustrating the roles of CBP and Ku70 maintaining a balanced ROS-associated redox in melanoma cells is presented in Fig. 8. In Fig. 8, CBP was proposed to play roles in producing antioxidants through the Nrf2-Keap1 pathway and also in switching off the expression of NOX2 through working with Ku70, offering the melanoma cells an appropriate redox condition for proliferation (Fig. 8). In such a process, CBP was also required by the expression of Ku70, which produces enough Ku70 to form the Ku70-BAX complex, to switch off the BAX-associated apoptotic cell death (the intrinsic pathway of apoptosis, Fig. 8). Obviously, CBP, Ku70, NOX2, and CBP, Ku70, and BAX are organized into lines of the regulatory network in balancing the oxidative stress for melanoma cells to proliferate (Fig. 8).

$\mathrm{Ku} 70$ and Ku80 have long been known to play roles in NHEJ repair of DNA DSBs ${ }^{31,32}$. During which, CBP/p300 promotes the recruitments of Ku70 and Ku80 to the DNA DSB site $^{33}$. Recently, the role of Ku70 in controlling apoptotic cell death has been found in NB cells. Ku70 binds to BAX to form the Ku70-BAX complex in the cytoplasm, which is found to be critical in controlling the intrinsic pathway of apoptosis. CBP could abolish the BAX binding capacity of Ku70 by acetylation modification on Ku70, releasing the BAX for initiating apoptosis via mitochondria ${ }^{13}$. In addition, similar acetylation modification of Ku80 by CBP was also found to be required by COX2 transcription $^{34}$. Moreover, Ku80 was found to be able to inhibit melanoma cell proliferation and induce apoptosis; however, similar roles of Ku70 in melanoma cells has remained unknown ${ }^{35}$. In this work, we have revealed two roles of Ku70 in melanoma cells: negatively regulate the transcription of NOX2 and positively control the apoptosis via forming Ku70-BAX complex. We clearly demonstrated that knocking down Ku70 in melanoma cells have elevated the ROS level, arrested cell cycle in the $\mathrm{S}$ phase, inhibited the proliferation of melanoma cells, and induced cell death, such as paraptosis, necrosis, and apoptosis (Fig. 8).

\section{Summary}

In this study, we found for the first time that $\mathrm{CBP}$ and Ku70 have been utilized in the negative regulation of NOX2 expression. Knocking down either CBP or Ku70 results in enhanced expression of NOX2, and increased 


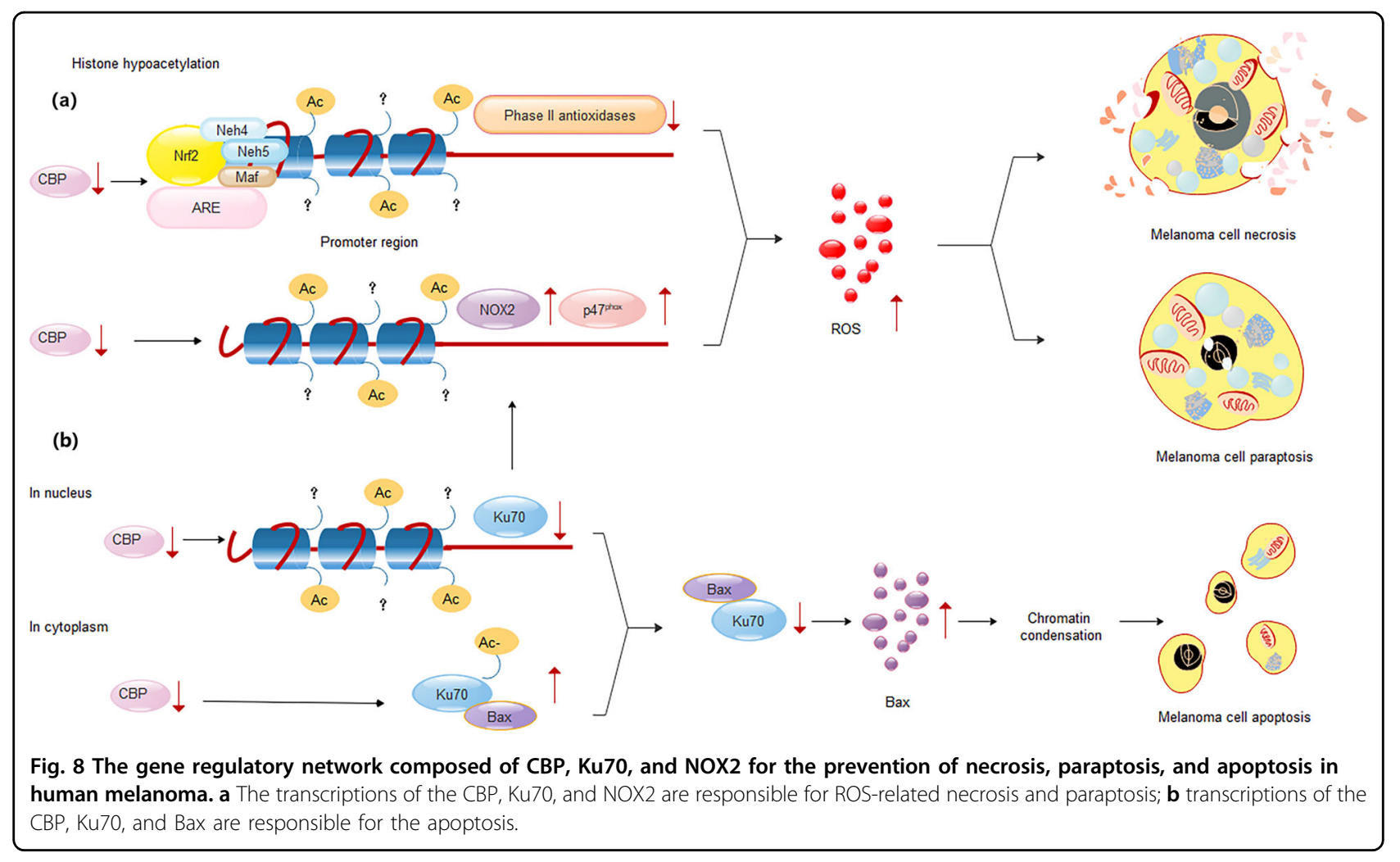

level of intracellular ROS, which is responsible for cytoplasmic vacuolization, cell cycle arrest in $\mathrm{S}$ phase, necrosis, and parapotic cell death. In addition, CBP also functions in the expression and the acetylation modification of Ku70. Knocking down CBP increased the level of BAX in the cytoplasm, leading to the release of proapoptotic factors from mitochondria and the activations of caspases for the induction of apoptosis. In summary, we found in this work that CBP, Ku70, NOX2, BAX, and caspase have been organized into a transcriptional network in balancing the ROS-related intracellular oxidative environments, which benefits the proliferation of melanoma cells by avoiding the necrosis, paratosis and apoptotic cell death.

\section{Materials and methods Materials}

Dulbecco's modified Eagle's medium (DMEM), penicillin/streptomycin, and fetal bovine serum (FBS) were purchased from Gibco-BRL (Gaithersburg, MD, USA). ExFect ${ }^{\mathrm{TM}} 2000$ transfection reagent was purchased from Vazyme Biotech (Nanjing, China). PrimeScript ${ }^{\mathrm{TM}}$ RT reagent kit (Perfect Real-Time) and SYBR Premix Ex Taq ${ }^{\text {TM }}$ II (Tli RNaseH Plus) were purchased from Takara Biotechnology Co. Ltd (Dalian, China). Trizol reagent was purchased from Invitrogen (Carlsbad, CA, USA). RIPA lysis buffer with a protease inhibitor phenylmethanesulfonyl fluoride (PMSF), PVDF membrane,
BCA protein assay kit, and ECL Western Blotting Substrate Kit were purchased from Solarbio (Beijing, China). Ku70 (acetyl L542) antibody (Rabbit IgG) was purchased from SAB (MD, USA). BAX antibody (Rabbit IgG) was purchased from ABcam (Cambridge, MA, USA). NOX2 antibody (Rabbit IgG), Ku70 antibody (Rabbit IgG), $\beta$-actin antibody (Rabbit IgG), and Goat Anti-Rabbit IgG $(\mathrm{H}+\mathrm{L})$ were purchased from ABways (Shanghai, China). Kit for analyzing caspase-3 activity was purchased from Beyotime Biotechnology Co. Ltd (Nantong, China). CCK-8 was purchased from Dojindo Molecular Technologies, Inc. (Kumamoto, Japan). Annexin V-propidium iodide (PI) apoptosis detection kit was purchased from Vazyme Biotech (Nanjing, China). Kits for analyzing the cell cycle and apoptosis were purchased from Beyotime Biotechnology Co. Ltd (Nantong, China). Antifade Mounting medium with DAPI was purchased from Solarbio (Beijing, China). Actinomycin D was purchased from the Sigma Chemical Co. (St. Louis, MO, USA). siRNAs (listed in Table 1) were designed by use of an online tool (http://biodev. extra.cea.fr/DSIR/DSIR.html), and examined for the sequence specificity by running Basic Local Alignment Search Tool [National Center for Biotechnology Information (NCBI)], and synthesized by Sangon Biotech (Shanghai, China). Primers used in this study are listed in Table 2, they were also synthesized by Sangon Biotech (Shanghai, China). 
Table 1 The sequences for siRNA.

\begin{tabular}{lll}
\hline siRNA & & Sequences \\
\hline CBP siRNA & Forward & GGCGAAUGACAGCACAGAUTT \\
& Reverse & AUCUGUGCUGUCAUUCGCCTT \\
Ku70 siRNA & Forward & GCAGUGGACCUGACAUUGCTT \\
& Reverse & GCAAUGUCAGGUCCACUGCTT \\
Nrf2 siRNA & Forward & GGAUUUGAUUGACAUACUUTT \\
& Reverse & AAGUAUGUCAAUCAAAUCCTT \\
NC siRNA & Forward & UUCUCCGAACGUGUCACGUTT \\
& Reverse & ACGUGACACGUUCGGAGAATT \\
\hline
\end{tabular}

Table 2 The primer sequences for RT-PCR.

\begin{tabular}{lll}
\hline Gene & & Primer DNA sequences \\
\hline Human CBP & Forward & ATTGATAACGAGGTCCCTACCC \\
& Reverse & GCTCCGTCTTCATTTCCAG \\
Human Ku70 & Forward & TCTCAAGCCTCCTCCAATA \\
& Reverse & GTCATCCAACTCTTCTTCCT \\
Human NOX2 ${ }^{20}$ & Forward & GCAGCCTGCCTGAATTCA \\
& Reverse & TGAGCAGCACGCACTGGA \\
Human $\beta$-actin & Forward & CCTGGCACCCAGCACAAT \\
& Reverse & GGGCCGGACTCGTCATAC \\
\hline
\end{tabular}

\section{Cell culture}

The human malignant melanoma A375 cell line (A375-P) was purchased from the Institute of Basic Medical Sciences, Chinese Academy of Medical Sciences (Beijing, China). The A375 cells were cultured in DMEM supplemented with $10 \% \mathrm{FBS}, 100 \mathrm{U} / \mathrm{ml}$ penicillin, and $100 \mathrm{mg} /$ ml streptomycin with $5 \% \mathrm{CO}_{2}$ at $37^{\circ} \mathrm{C}$ in a humidified incubator $^{36,37}$. All manipulations were carried out by strictly following the experimental guidelines and ethical regulations of Hebei University (Baoding, China).

\section{Transfection of siRNA}

Transfections of siRNA into melanoma cells were performed by use of an ExFect ${ }^{\mathrm{TM}} 2000$ Transfection Reagent. The cells were seeded by $1 \times 10^{5}$ cells/well into the wells in six-well plates. A375 cells were transfected with siRNAs treated with the transfection reagent when the cells grow $\sim 60-80 \%$ in the wells. The siRNAs were diluted into different concentrations using a serum-free medium and mixed up with an equal volume of transfection reagents before use $\mathrm{s}^{38}$. Melanoma cells were subdivided into control groups, NC siRNA group, and experimental groups. Transfections of the experimental group with siRNA were carried out by a concentration of 1,2 , and $3 \mathrm{fmol} / \mathrm{cell}$, respectively, (the depletion efficiencies were scored to be equal to $70 \%$ of the Ku70 mRNA and the CBP mRNA to that in the control group, respectively, for the single transfections with either CBP siRNA or Ku70 siRNA, and to $60 \%$ of CBP mRNA and Ku70 mRNA to that in the control group for co-transfections of the CBP siRNA and Ku70 siRNA, respectively). The transfected A375 cells were incubated for $24 \mathrm{~h}$ before analysis. The depletion efficiencies were scored by RT-qPCR following the protocol from the manufacture.

\section{RNA extraction and real-time quantitative reverse transcription PCR}

Total RNA molecules were prepared using Trizol reagent by following the standard protocol from the manufacturer $^{39}$. RNA samples were reverse transcribed into cDNA using a PrimeScript ${ }^{\mathrm{TM}}$ RT reagent kit with gDNA eraser (TaKaRa Biotechnology Co. Ltd) in a total volume of $20 \mu \mathrm{l}$ by following the manufacturer's proto$\mathrm{col}^{40}$. The cDNA molecules obtained by the above procedure were used as amplification templates, and the expression levels of the target genes were measured by real-time quantitative PCR. The PCR was quantified according to the instructions of the SYBR Premix Ex Taq TM II (Tli RNaseH Plus) kit (Takara Biotechnology Co. Ltd) in a CFX96 Real-Time PCR Detection System. The amplification steps were as follows: steps are (i) at $95^{\circ} \mathrm{C}$ for $30 \mathrm{~s}$; (ii) 40 cycles at $95^{\circ} \mathrm{C}$ for $5 \mathrm{~s}$; (iii) at $55^{\circ} \mathrm{C}$ for $30 \mathrm{~s}$; and (iv) extension at $72{ }^{\circ} \mathrm{C}$ for $30 \mathrm{~s}$. $\beta$-actin was used as an endogenous reference, and each sample was normalized to its $\beta$-actin content. Results are represented as the refractive induction using the $2^{-\triangle \triangle \mathrm{Ct}}$ method.

\section{Protein extraction and western blot analysis}

Cells were harvested and lysed using a RIPA lysis buffer (Solarbio, Beijing, China) with PMSF (Solarbio, Beijing, China) by following the manufacturer's instruction. Protein concentrations were determined by using a BCA protein assay kit (Solarbio, Beijing, China). An equal amount of protein was loaded onto different wells on an SDS-PAGE gel (containing 7.5\% SDS), and electrophoresis was performed at a constant voltage of 120 volts. The separated proteins were then transferred to a PVDF membrane (Solarbio, Beijing, China) at a constant current of 280 amps. The membrane was blocked with $5 \% \mathrm{w} / \mathrm{v}$ skim milk for $1 \mathrm{~h}$ at room temperature. The membrane was then incubated in solutions containing either NOX2 antibody (1:1000 dilution), Ku70 antibody (1:500 dilution), or $\beta$-actin antibody (1:1000 dilution) at $4{ }^{\circ} \mathrm{C}$ for an overnight. Goat anti-rabbit IgG $(\mathrm{H}+\mathrm{L})$ (1:1000 dilution) coupled with the corresponding horseradish peroxidase was incubated with an ECL Western Blotting substrate kit. The protein bands were observed and repeated three 
times for each of the samples. The ImageJ software was used to quantify the bands and to normalize the target protein level to $\beta$-actin.

The relative expression of the target protein $=$ the target protein ID value/the internal reference ID value.

\section{CCK-8 assay}

Melanoma A375 cells of $5 \times 10^{3}$ cells/well were seeded in each well in a 96-well plate and incubated for $24 \mathrm{~h}$. Then CCK-8 solution was added to each well, and the cells further incubated at $37^{\circ} \mathrm{C}$ for $60 \mathrm{~min}$. Cell viability was measured by the absorbance at $450 \mathrm{~nm}$ on a microplate reader ${ }^{37}$. The cell proliferation rates were calculated by use of the following equation:

Cell proliferation rates $=\mathrm{A}$ (transfection group) $/ \mathrm{A}$ (control group) $\times 100 \%$.

\section{Detection of intracellular ROS}

The ROS in the melanoma A375 cells were determined using a ROS assay kit purchased from Beyotime Institute of Biotechnology (Haimen, Jiangsu, China). The fluorescent probe used in the assay was $2^{\prime}, 7^{\prime}$-dichlorofluorescein diacetate (DCFH-DA). DCFH-DA could diffuse into cells and be oxidized by ROS and converted into DCFH, which can be further oxidized to a fluorescent product DCF by ROS.

For the determination of the intracellular ROS in the transfected A375 cells, $10 \mathrm{mM}$ DCFH-DA was diluted to a final concentration of $10 \mu \mathrm{M}$ with a serum-free DMEM medium. In $24 \mathrm{~h}$ of siRNA transfection, the original cell culture medium was discarded and $1 \mathrm{ml}$ of DCFH-DA solution $(10 \mu \mathrm{M})$ was added into each well, and the cells were further incubated at $37^{\circ} \mathrm{C}$ for $30 \mathrm{~min}$ and then washed three times using DMEM medium ${ }^{41}$. The ROS of the cell suspension was analyzed at excitation wavelength $488 \mathrm{~nm}$ and emission wavelength $525 \mathrm{~nm}$, using FACS can flow cytometer (BD Biosciences).

\section{Cell death assay}

Cell death caused by the transfections of CBP siRNA and Ku70 siRNA was analyzed by use of a kit containing $\mathrm{PI}$ and Annexin V-FITC. Firstly, the transfected cells were cultivated at $37^{\circ} \mathrm{C}$ with $5 \% \mathrm{CO}_{2}$ for 24 and $48 \mathrm{~h}$, respectively, and then they were harvested and digested into single-cell suspensions using trypsin digestion solutions (0.25\%, without phenol red; Solarbio, Beijing, China). The single cells were washed twice with cold PBS and resuspended in $100 \mu$ l of $1 \times$ binding buffer (supplied by the manufacturer). The cells were double-stained in dark at room temperature by mixing up with $5 \mu \mathrm{lo}$ PI staining solution and $5 \mu \mathrm{l}$ of Annexin V-FITC for $10 \mathrm{~min}$, and resuspended in $400 \mu \mathrm{l}$ of $1 \times$ binding buffer (supplied by the manufacturer). The cell death was analyzed within $1 \mathrm{~h}$ by a FACS Calibur instrument (FACS Calibur, BD, USA; the excitation wavelength was $488 \mathrm{~nm}$, and the AnnexinFITC and PI were measured in FL1 channel, and in FL2 or FL3 channel, respectively) ${ }^{42}$.

\section{Observation of cytoplasm vacuolization}

The human melanoma A375 cells were seeded into the wells of a six-well plate by $1 \times 10^{5}$ cells per well and cultivated for $24 \mathrm{~h}$ (as described in the previous). Transfections for knocking down CBP mRNA and Ku70 mRNA individually or simultaneously in the melanoma A375 were conducted, using CBP siRNA and Ku70 siRNA (as described in the "Transfection of siRNA" section). The transfected cells were further cultured for $24 \mathrm{~h}$, and the morphological changes of the cells were observed by using an optical microscope at an interval of $8 \mathrm{~h}$.

For the observations of the effects of actinomycin D on cytoplasm vacuolization in the cells transfected with CBP siRNA, melanoma cells were seeded in a six-well plate by $1 \times 10^{5}$ cells per well and cultivated for $24 \mathrm{~h}$. CBP siRNA (70\% efficiency of depletion) and actinomycin D $(5 \mu \mathrm{g} / \mathrm{ml})$ were added simultaneously, and the cell cytoplasm vacuolization was observed under a light microscope in $24 \mathrm{~h}$ of transfection.

\section{Chromatin condensation analysis}

The chromatin condensation was analyzed using DAPI staining $^{43}$, specifically, $1 \times 10^{5}$ melanoma A375 cells were seeded into a well in a six-well plate placed with a sterilized glass coverslip in the well, and cultivated in the presence of $5 \% \mathrm{CO}_{2}$ gas at $37^{\circ} \mathrm{C}$ for $24 \mathrm{~h}$. The cells were subdivided into control group, CBP siRNA group (with $70 \%$ efficiency of CBP mRNA depletion), Ku70 siRNA group (with $70 \%$ efficiency of Ku70 mRNA depletion), CBP-Ku70 siRNA co-transfection group (with 60\% efficiencies of CBP mRNA and Ku70 mRNA depletion, respectively), and NC siRNA group. The cells of each group were processed using $1 \mathrm{ml}$ fix solution containing 4\% paraformaldehyde (Beyotime Biotechnology, Nantong, China) for $10 \mathrm{~min}$, when the cultivations were at $0,8,12$, 16,20 , and $24 \mathrm{~h}$, respectively. The fixed cells in each well were washed twice with PBS, and then further processed by adding $1 \mathrm{ml}$ of Immunostaining Permeabilization Buffer containing Triton X-100 (Beyotime Biotechnology, Nantong, China) into the wells for $10 \mathrm{~min}$ at room temperature. The cells were then stained in $10 \mu \mathrm{l}$ of Antifade mounting medium with DAPI (Solarbio, Beijing, China). The chromatin condensation was analyzed under a fluorescence microscope (Olympus, BX61; ×1000).

\section{Cell cycle analysis}

A375 cells were seeded by $1 \times 10^{5}$ into each well containing $2 \mathrm{ml}$ of DMEM medium in a six-well plate and cultivated for $24 \mathrm{~h}$ to allow cells to adhere. After $8 \mathrm{~h}$ of transfection, fresh DMEM was added to replace the 
used DMEM medium, and further cultivated for $24 \mathrm{~h}$. Cells were then harvested and trypsinized (trypsin digestion solutions, $0.25 \%$ phenol red free) into a singlecell suspension. The single cells were washed twice with PBS and then fixed using $70 \%$ ethanol at $4{ }^{\circ} \mathrm{C}$ overnight. The fixed cells were then washed once with PBS and then $1 \mathrm{ml}$ of PI staining reagent (containing $50 \mathrm{mg} / \mathrm{ml} \mathrm{PI}$ and $1 \mathrm{mg} / \mathrm{ml}$ RNase in $1 \mathrm{ml}$ of sodium citrate buffer, $\mathrm{pH}$ 7.4) was added and kept in dark at room temperature for $30 \mathrm{~min}$ (ref. ${ }^{44}$ ). The samples were analyzed by flow cytometry on a FACSC alibur apparatus (FACS Calibur, $\mathrm{BD}$, USA). Cells untreated were used as controls. Quantitation of cell cycle distributions was performed using Multi-cycle Software (ModFit software). The percentage of cells in G1, S, and G2 phases was calculated, respectively.

\section{Measurements of caspase- 3 activity}

Activities of caspase- 3 were analyzed using a detection kit (Beyotime, China). Briefly, $2 \times 10^{6}$ A375 cells were collected in a $2 \mathrm{ml}$ tube and digested using trypsin. Supernatants were discarded after the cells were centrifuged at $4{ }^{\circ} \mathrm{C}(2000 \times g$ for $5 \mathrm{~min})$. The cells harvested were then washed once using PBS and lysed in $100 \mu \mathrm{l}$ lysis buffer on ice for $20 \mathrm{~min}$. The caspase- 3 activities in the cell lysates were measured by mixing up the cell lysates with $40 \mu \mathrm{l}$ of detection buffer and $10 \mu \mathrm{l}$ of Ac-DEVD-pNA $(2 \mathrm{mM})$, respectively, and incubating the mixtures at $37^{\circ} \mathrm{C}$ for $120 \mathrm{~min}$. The absorbance at $405 \mathrm{~nm}$ was monitored using a microplate Reader.

\section{Statistical analysis}

All data are presented as means \pm SEM in this work. Statistical significance was determined by using the oneway ANOVA for multiple comparisons, and the Student's $t$ test of the SPSS22.0 software for comparing the mean differences between every two groups. The data were considered to be significant when $P<0.05$. All experiments were performed in triplicate.

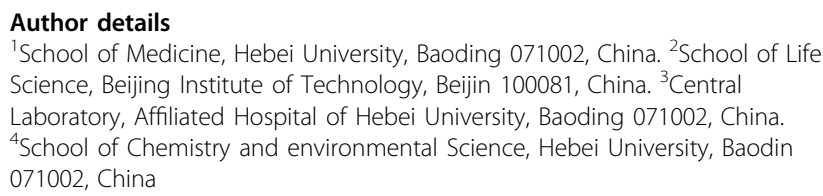

\section{Funding}

This work has been partly supported by Beijing Natural Science Foundation (No. 5132014 to X.P.), Hebei provincial key Research Programs for Medical Science (No. 20160051 to X.P.), and the Collaborative Innovation Project for Drug Development between Beijing, Tianjin, and Hebei Province, China (S.S. and X.P.) and the Key Project of Baoding Lianchi District Science and Technology Bureau, China, (No. 15J04 to L.D. and X.P.).

Conflict of interest

The authors declare no competing interests.

\section{Publisher's note}

Springer Nature remains neutral with regard to jurisdictional claims in published maps and institutional affiliations.

Received: 22 February 2020 Revised: 3 January 2021 Accepted: 3 February 2021

Published online: 26 February 2021

\section{References}

1. Alam, M. M. et al. Glucocorticoid receptor signaling represses the antioxidant response by inhibiting histone acetylation mediated by the transcriptional activator NRF2. J. Biol. Chem. 292, 7519-7530 (2017).

2. Hartman, M. L. \& Czyz, M. MITF in melanoma: mechanisms behind its expression and activity. Cell Mol. Life Sci. 72, 1249-1260 (2015).

3. Mukherjee, S. P. et al. Analysis of the RelA:CBP/p300 interaction reveals its involvement in NF-kB-driven transcription. PLoS Biol. 11, e1001647 (2013).

4. Diefenbacher, M. E. et al. The LIM domain protein nTRIP6 recruits the mediator complex to AP-1-regulated promoters. PLOS ONE 9, e97549 (2014).

5. Tanigawa, S., Fujii, M. \& Hou, D. X. Action of Nrf2 and Keap1 in AREmediated NQO1 expression by quercetin. Free Radic. Biol. Med. 42, 1690-1703 (2007).

6. Zhang, J. et al. Nrf2 Neh5 domain is differentially utilized in the transactivation of cytoprotective genes. Biochem J. 404, 459-466 (2007).

7. Subramanian, C., Jarzembowski, J. A., Opipari, A. W. Jr, Castle, V. P. \& Kwok, R. P. CREB-binding protein is a mediator of neuroblastoma cell death induced by the histone deacetylase inhibitor trichostatin A. Neoplasia 9, 495-503 (2007).

8. Giralt, A. et al. Long-term memory deficits in Huntington's disease are associated with reduced CBP histone acetylase activity. Hum. Mol. Genet. 21, 1203-1216 (2011).

9. Rouaux, C., Loeffler, J. P. \& Boutillier, A. L. Targeting CREB-binding protein (CBP) loss of function as a therapeutic strategy in neurological disorders. Biochem. Pharmacol. 68, 1157-1164 (2004).

10. Jung, J. \& Bonini, N. CREB-binding protein modulates repeat instability in a drosophila model for polyQ disease. Science 315, 1857-1859 (2007).

11. Zhao, Y. et al. Corepressive action of CBP on androgen receptor transactivation in pericentric heterochromatin in a drosophila experimental model system. Mol. Cell Biol. 29, 1017-1034 (2009).

12. Manickavinayaham, S. et al. E2F1 acetylation directs p300/CBP-mediated histone acetylation at DNA double-strand breaks to facilitate repair. Nat. Commun. 10, 4951 (2019).

13. Subramanian, C., Hada, M., Opipari, A. J., Castle, V. P. \& Kwok, R. P. CREB-binding protein regulates $\mathrm{Ku} 70$ acetylation in response to ionization radiation in neuroblastoma. Mol. Cancer Res. 11, 173-181 (2013).

14. Hada, M., Subramanian, C., Andrews, P. C. \& Kwok, R. P. Cytosolic Ku70 regulates Bax-mediated cell death. Tumour Biol. 37, 13903-13914 (2016).

15. Mazumder, S., Plesca, D., Kinter, M. \& Almasan, A. Interaction of a cyclin E fragment with Ku70 regulates Bax-mediated apoptosis. Mol. Cell Biol. 27, 3511-3520 (2007)

16. Kim, B. \& Chung, M. H. W. Hypoxia/reoxygenation induces apoptosis through a ROS-mediated caspase-8/Bid/Bax pathway in human lymphocytes. Biochem. Biophys. Res. Commun. 363, 745-750 (2007).

17. Pérez, E., Lindblad, J. L. \& Bergmann, A. Tumor-promoting function of apoptotic caspases by an amplifi.cation loop involving ROS, macrophages and JNK in drosophila. Elife 6, e26747 (2017).

18. Fu, X. F. et al. PGC-1a regulates the cell cycle through ATP and ROS in CH1 cells. J. Zhejiang Uni. Sci. B 17, 136-146 (2016).

19. Bradshaw, P. C. Cytoplasmic and mitochondrial NADPH-coupled redox systems in the regulation of aging. Nutrients 11, 504 (2019).

20. Chen, F. et al. Inhibition of histone deacetylase reduces transcription of $\mathrm{NADPH}$ oxidases and ROS production and ameliorates pulmonary arterial hypertension. Free Radic. Biol. Med. 99, 167-178 (2016).

21. Chen, T. S. et al. Taxol induces caspase-independent cytoplasmic vacuolization and cell death through endoplasmic reticulum (ER) swelling in ASTC-a-1 cells. Cancer Lett. 270, 164-172 (2009).

22. Engelbrecht, D., Durand, P. M. \& Coetzer, T. L. On programmed cell death in plasmodium falciparum: status quo. J. Trop. Med. 2012, 646534 (2012).

23. Leung, $\mathrm{C}$. H. et al. Emerging screening approaches in the development of Nrf2-Keap1 protein-protein interaction inhibitors. Int. J. Mol. Sci. 20, 4445 (2019). 
24. Juhasz, A. et al. Expression of NADPH oxidase homologues and accessory genes in human cancer cell lines, tumours and adjacent normal tissues. Free Radic. Res. 43, 523-532 (2009).

25. Li, Hanjie et al. Dysfunctional CD8 T cells form a proliferative, dynamically regulated compartment within human melanoma. Cell 176, 775-789 (2019).

26. Meza, C. A., La, Favor, J. D., Kim, D. H. \& Hickner, R. C. Endothelial dysfunction: is there a hyperglycemia-induced imbalance of NOX and NOS? Int. J. Mol. Sci. 20, 3775 (2019).

27. NavaneethaKrishnan, S., Rosales, J. L. \& Lee, K. Y. ROS-Mediated cancer cell killing through dietary phytochemicals. Oxid. Med. Cell Longev. 2019, 9051542 (2019).

28. Zhang, M. et al. Emerging roles of Nrf2 and phase $\|$ antioxidant enzymes in neuroprotection. Prog. Neurobiol. 100, 30-47 (2013).

29. Manea, S. A., Constantin, A., Manda, G., Sasson, S. \& Manea, A. Regulation of Nox enzymes expression in vascular pathophysiology: focusing on transcription factors and epigenetic mechanisms. Redox Biol. 5, 358-366 (2015).

30. Choudhari, S. K., Chaudhary, M., Bagde, S., Gadbail, A. R. \& Joshi, V. Nitric oxide and cancer: a review. World J. Surg. Oncol. 11, 118 (2013).

31. Mouw, K. W., Goldberg, M. S., Konstantinopoulos, P. A. \& D'Andrea, A. D. DNA damage and repair biomarkers of immunotherapy response. Cancer Discov. 7 675-693 (2017).

32. Lieber, M. R. The mechanism of double-strand DNA break repair by the nonhomologous DNA end-joining pathway. Annu. Rev. Biochem 279, 181-211 (2010).

33. Reed, S. M. \& Quelle, D. E. p53 acetylation: regulation and consequences. Cancers 7, 30-69 (2014).

34. Xiao, Y. et al. Ku80 cooperates with CBP to promote COX-2 expression and tumor growth. Oncotarget 6, 8046-8061 (2015).
35. Liu, T. et al. Ku80 promotes melanoma growth and regulates antitumor effect of melatonin by targeting HIF1-a dependent PDK-1 signaling pathway. Redox Biol. 25, 101197 (2019).

36. Zhu, X., Li, W. \& Meng, Q. LncRNA H19 promotes proliferation and invasion in A375 human melanoma cell line. Int. J. Clin. Exp. Pathol. 11, 1063-1073 (2018).

37. Herwig, N., Belter, B., Wolf, S., Haase-Kohn, C. \& Pietzsch, J. Interaction of extracellular S100A4 with RAGE prompts prometastatic activation of A375 melanoma cells. J. Cell Mol. Med. 20, 825-835 (2016).

38. Sun, $\mathrm{X}$. et al. The Nrf2 activator RTA-408 attenuates osteoclastogenesis by inhibiting STING dependent NF-kb signaling. Redox Biol. 28, 101309 (2019).

39. Lai, Y., Xu, X., Zhu, Z. \& Hua, Z. Highly efficient siRNA transfection in macrophages using apoptotic body-mimic Ca-PS lipopolyplex. Int. J. Nanomed. 13, 6603-6623 (2018).

40. Cui, L., Hu, H., Wei, W., Wang, W. \& Liu, H. Identification and characterization of microRNAs in the liver of blunt snout bream (megalobrama amblycephala) infected by aeromonas hydrophila. Int. J. Mol. Sci. 17, 1972 (2016).

41. Cui, L. et al. Molecular cloning, characterization and expression profiling of a ryanodine receptor gene in Asian corn borer, Ostrinia furnacalis (Guenée). PLoS ONE 8, e75825 (2013).

42. Wang, $C$. et al. Manganese increases the sensitivity of the CGAS-STING pathway for double-stranded DNA and is required for the host defense against DNA viruses. Immunity 17, 675-687 (2018).

43. Zhang, Z. et al. Tauroursodeoxycholic acid alleviates secondary injury in the spinal cord via up-regulation of CIBZ gene. Cell Stress Chaperones 23, 551-560 (2018).

44. Kaium, M. A. et al. H2S donor, S-propargyl-cysteine, increases CSE in SGC-7901 and cancer-induced mice: evidence for a novel anti-cancer effect of endogenous H2S? PLOS ONE 6, e20525 (2011). 